\title{
A Distributed Fault-Detection and Diagnosis System Using On-Line Parameter Estimation
}

T.-H. Guo and W. Merrill

Lewis Research Center

Cleveland, Ohio

and

\section{A. Duyar}

Florida Atlantic University

Boca Raton, Florida

Prepared for the

International Symposium on Distributed Intelligence Systems

sponsored by the International Federation of Automatic Control

Technical Committee on Systems Engineering

Arlington, Virginia, August 13-15, 1991

\section{N/Sก}




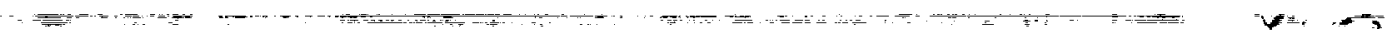




\title{
T.-H. Guo and W. Merrill
}

National Aeronautics and Space Administration, Lewis Research Center, Cleveland, Ohio, USA

\author{
A. Duyar \\ Department of Mechanical Engineering, Florida Atlantic University, Boca Raton, Florida, USA
}

\begin{abstract}
This paper describes a model-based fault-detection and diagnosis system based on a distributed system identification approach. The diagnostic system consists of a two level process including parallel hypothesis testing modules and a fault mode identification and estimation module. The proposed system is part of a distributed diagnostic system for use in an intelligent control system. The proposed approach utilizes a piecewise linear model to predict the system performance. The deviation between predicted and actual performance is used to identify the associated fault mode. Each hypothesis testing module is associated with a particular class of fault modes and can be viewed as a condition monitor in a distributed diagnostic system hierarchy. The results of the hypothesis modules are processed by the fault-detection and estimation module. Using the results of the on-line diagnosis, the intelligent control system will be able to accommodate the fault modes, reduce maintenance cost, and increase system availability.
\end{abstract}

\section{INTRODUCTION}

There is a growing demand to improve the control of systems for enhanced performance with increased reliability, durability and maintainability. This demand can be met by improving the individual reliability of system components and also by an intelligent control system with fault-detection, diagnostics and accommodation capabilities $[1,2]$. This paper focuses on the development of a model-based fault-detection and diagnosis (FDD) system which can be used as an integral part of such an intelligent control system.

During the last two decades of the development of faultdetection methods, the so called model-based fault-detection approach has received considerable attention $[3,4,5,6]$. These schemes basically rely on the idea of analytical redundancy. As opposed to physical redundancy which uses measurements from redundant sensors for fault-detection purposes, analytical redundancy is based on the signals generated by the mathematical model of the system being considered. These signals are then compared with the actual measurements obtained from the system. The residual quantities are generated by comparing the measured and the model-generated signals. Hence, the modelbased fault-detection and diagnosis is defined as the determination of faults of a system from the comparison of the measurements of the system with a priori information represented by the model of the system.

A fault is defined as a malfunction that deteriorates a plant's ability to perform its specified tasks. Since the faults alter the system dynamics, they can be modelled as changes in the system's parameters. The fault-detection task is the act of identifying the existence of these changes. The fault diagnosis task is the act of isolating and estimating the magnitude of the fault. The basis for the isolation of a fault is the fault signature, i.e. a signal obtained from a diagnostic model defining the effects associated with a class of faults. A diagnostic model is obtained by defining the residual vector in such a manner that its direction is associated with known fault signatures. Furthermore, each signature has to be unique to one fault in order to accomplish fault isolation. A set of parity relations [3] or a set of unknown input observers [4], each assigned to be sensitive to a different fault, can be used for this purpose.

The organization of this paper is as follows. First, the method of modelling a complex system will be described. This is followed by a description of diagnosis models which include process faults. Next, the architecture for fault-detection and diagnosis is described. Finally, simulation results of fault diagnosis of the Space Shuttle Main Engine (SSME) are given.

\section{PROCESS MODEL}

The nominal condition of a process under study can be modelled as a discrete, linear, time-invariant system described by:

$$
\begin{aligned}
x(n+1) & =A x(n)+B u(n) \\
y(n) & =C x(n)
\end{aligned}
$$

where $\mathrm{x}$ is the state vector, $\mathrm{u}$ is the input vector and $\mathrm{y}$ is the output vector.

The matrices $\mathrm{A}, \mathrm{B}$, and $\mathrm{C}$ of this model can be determined by using a multivariable system identification technique. A system identification algorithm, developed in [7] to determine these parameters based on the observability indices of the system from the measurements of the input and output data, was used in this paper. The A, B, C matrices obtained for this model will be used as baseline process parameters of the system. Any changes of these parameters observed through real time identification, away from preselected threshold values are used to detect and diagnose the faults.

Furthermore, if the system is to be operated over a wide range and a linear model can not accurately represent the system characteristics then a series of parameter identifications will be needed to cover the possible range of operation conditions. A piecewise linear model which links all the 
operation conditions can be described by:

$$
\begin{aligned}
x(n+1) & =A\left(y_{s}\right) x(n)+B\left(y_{s}\right) u(n) \\
y(n) & =C\left(y_{y}\right) x(n)
\end{aligned}
$$

where $y_{\mathrm{s}}$ is the scheduling variable and is a subset of the output measurement $\mathrm{y}$.

\section{MODELLING THE PROCESS FAULTS}

In general, there are three classes of fault modes covered by the system performance model of equation (1), namely actuator faults, sensor faults and system performance degradation. In this study, actuator faults are modelled by the changes of actuation gain matrix B. Sensor faults are modelled by the changes of observation matrix $C$. And, system performance degradations (dynamic changes) are modelled by the system characteristic matrix $A$. Under these assumptions, these fault modes can be isolated and diagnosed by analyzing the observed behavior through hypothesis testing which will be described latter

\section{Actuator Fault Model}

An actuator fault occurs when the actuator output cannot follow the command signal. The error can be either multiplicative or additive. It can be described by the following equation:

$$
u_{n f}(n)=F_{n} u_{c}(n)+f_{n o}
$$

where $u_{n f}(n)$ is the actual system input under the actuator fault condition and $u_{c}(n)$ is the commanded system input. $F$ is a diagonal matrix representing the multiplicative distortion of the command signal and $f_{\text {ao }}$ is a constant vector representing the bias, both with appropriate dimensions.

During nomal operation, $F_{a}=I$ and $f_{a 0}=\underline{0}$. Different fault modes will result in different values of $F_{a}$ and $f_{a 0}$. The values of $F$, and $f_{a o}$ will be estimated and used to identify the corresponding fault modes.

By replacing the input signal $u$ in equation (1) with the actual input signal $u_{a f}$ a model for the system with the actuator faults is obtained.

$$
\begin{aligned}
x(n+1) & =A x(n)+B F_{a} u_{c}(n)+B f_{n o} \\
y(n) & =C x(n)
\end{aligned}
$$

$B F$, is the new input gain matrix and $B f_{* 0}$ is a bias term.

\section{Sensor Fault Model}

Similar to the way actuator faults were handled, sensor faults can also be modelled as a combination of multiplicative and bias errors:

$$
y_{s f}(n)=F_{s} y(n)+f_{s o}
$$

where $y_{s i}(n)$ are the sensor outputs through possible failed sensors and $y(n)$ the actual process outputs. The matrix $F_{\text {, }}$ is a diagonal matrix for the multiplicative error and $f_{s o}$ is a constant vector for the measurement bias, both with appropriate dimensions. During nomal operation, $F_{1}=I$ and $f_{x 0}=\underline{0}$. This model can represent a wide range of sensor faults, such as calibration errors (one of the diagonal elements of $F_{1} \neq 1$ and/or $f_{20} \neq 0$ ), loss of signals (one of the diagonal element of $F$, is 0 ), drift $\left(f_{x 0} \neq \underline{0}\right)$, cross wiring $\left(F_{a} \neq I\right)$ and many others.

The system model of the process with failed sensors can be obtain as:

$$
\begin{gathered}
x(n+1)=A x(n)+B u(n) \\
y_{u}(n)=F, C x(n)+f_{n}
\end{gathered}
$$

\section{System Degradation Model}

In the case of system performance degradation, it is assumed that only the system matrix $A$ will be affected. The new system matrix under this fault condition becomes $A_{1}$. In general, the fault model can be represented by:

$$
A_{r}=A+\Delta A
$$

where $\Delta \mathrm{A}$ is a matrix representing the effect of the fault mode under study. The determination of the elements of $\triangle A$ requires the analysis of the system using a physical model or empirical data.

The process model of a system with performance degradation becomes:

$$
\begin{aligned}
x(n+1) & =(A+\Delta A) x(n)+B u(n) \\
y(n) & =C x(n)
\end{aligned}
$$

We now define $F_{s}, f_{s o}, F_{a}, f_{n o}$ and $\Delta A$ as fault parameters. The following section describes the strategy of detecting the fault and estimating the fault parameters using a distributed on-line parameter identification scheme.

For a complete model that describes all three possible classes of faults the system equation will be:

$$
\begin{aligned}
x(n+1) & =(A+\Delta A) x(n)+B F_{n} u_{c}(n)+B f_{a o} \\
y_{a f}(n) & =F_{0} C x(n)+f_{s o}
\end{aligned}
$$

\section{FAULT-DETECTION AND DIAGNOSIS}

In the fault-detection and diagnosis for the system modelled by equation (9), one approach is to have an on-line estimation algorithm for all fault parameters in the equation. The estimated fault parameters can be compared to the predetermined signature of the fault modes of different classes. This approach is difficult in estimating many fault parameters at the same time. Also, the signatures of the fault parameters can be ambiguous if they were estimated by a single module. Thus, instead of direct estimation of parameter matrices A, B, C, and their related fault parameters, a two-step approach is proposed. The first step composed of a group of "Hypothesis Testing Modules" (HTM) in parallel processing to test each class of faults. Each module is solely designed to process the input/output data under a specified hypothesis and generate the fault signature data for diagnostics purposes. The second step is the fault diagnosis module which checks all the information obtained from the HTM level, isolates the fault, and determine its magnitude. Figure 1 shows the structure of the fault detection and diagnostic system.

\section{Hypothesis Testing Modules}

As illustrated in Figure 1, there are three fault parameter estimation modules in the first data processing layer. These modules are used for on-line identification of fault parameters corresponding to hypothesized actuator, sensor or system faults. The first module process the data under the assumption of possible actuator faults, i.e. modelled by equation (4). The goal of this module is to estimate the actuator fault parameters $(F$. and $\left.f_{a o}\right)$ using the on-line input/output data $\left(u_{c}\right.$ and $\left.y\right)$ assuming system matrices $A, B$ and $C$ are known. Since the fault parameters are the only unknown in equation (4), they can be estimated by a recursive on-line parameter estimation algorithm. Likewise, the sensor fault hypothesis testing module uses equation (6) and the system degeneration testing module uses equation (8) to estimate their fault parameters. Upon the estimation of the fault parameters, it is also necessary to determine the validity of the hypothesis. This is accomplished 


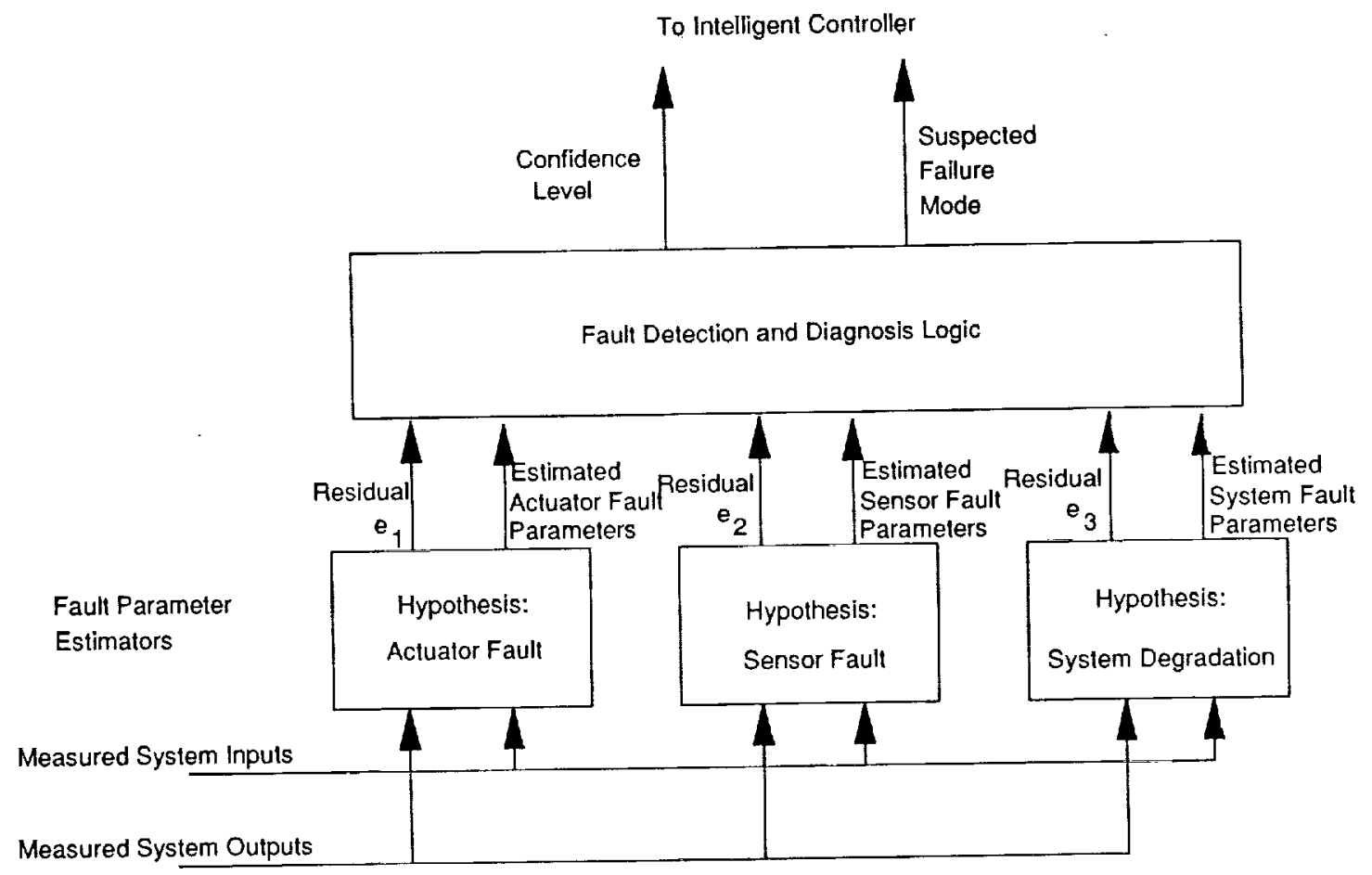

Figure 1 Distributed Model-Based Fault Detection and Diagnostic System

by comparing an output estimate obtained using the estimated fault parameters with the actual measured output. For this purpose the residual of the proposed model is clefined as:

$$
e_{v j}(n)=z_{i}(n)-\hat{y}_{1}\left(n / n-1, H_{j}\right)
$$

here subscript $i$ and $j$ refers to the $i$ th output and $j$ th class of faults. $z_{1}(n)$ is the measurement of $i$ 'th output. $H_{j}$ represents the hypothesis that the fault belongs to the $\mathrm{j}$ 'th class of faults. $\hat{y}_{1}\left(n / n-1, H_{1}\right)$ is the estimation of the $i$ th output given all the information up to $n-1$ 'th sampling under the hypothesis $H_{j}$. The values of $e_{1 \mid}$ are calculated at each step using the most recent estimate of the fault parameters and the statistics of $e_{i j}$ are used to accept or reject the hypothesis.

\section{Fault Detection and Diagnosis Logic}

This module examines all the estimated fault parameter values and the statistics of the residual vectors and generates a conclusion as to the fault status of the system. This is done by 1) comparing statistics of the residual vectors against preselected thresholds, 2) comparing the fault parameters against predetermined signatures, and 3) comparing the relative magnitude of the statistics of the residual vectors among all the hypothesis testing modules. By examining the relative magnitudes of the residual vectors from the different hypothesis modules we are able to detect the fault, classify the fault type, and estimate it magnitude. For example, when operating with an actuator fault, it is expected that the magnitude of the residual generated by the first hypothesis module (assuming an actuator fault) will be significantly smaller than those generated by other hypothesis modules. Also, the estimated fault parameters $F_{n}$ and $f_{\text {an }}$ will give the indication of the type of actuator faults.

Once a fault is detected, it may be isolated to the component that has failed by comparing the fault parameters with the known signatures of the fault modes. Measures can then be taken to compensate for the fault through reconfigura tion [2]. This diagnosis-induced acconmodiation includes hoth hardware actions (e.g., activating hack-up systems) and software tasks (e.g., adjusting the feedhack control appropriately, or estimating the measurement of a failed sensor). The diagnostic and monitoring tasks may be carried nut by an onboard processor, on-line and in real-time, as well as an off-line processor which analyzes recorded data for life cycle analysis and preventive maintenance.

\section{AN EXAMPLE: FAULT-DETECTION AND DIAGNOSIS OF THE SSME}

The fault-detection and diagnosis (FDD) system based on fault paraneter estimation, developed in this study was applied to the detection and diagnosis of the actuator and sensor faults for the space shutule main engine (SSME). A linearized model of the SSME nominal operation is given in $[9,10]$. A piecewise linear model which covers a wide range of operation was developed in 111$]$. The system parameters developed in $[10,11]$ is used as a priori knowledge for the FDD system.

The signature of a fault mode can usually be obtained through the analysis of physical property or empirical data. In the Space Shuttle Main Engine study, the commony observed actuator faults can be classified into four types: valve ball seal leakage or crack, valve line blockage, stuck valve and loss of rotational variable displacement transformer (RVDT) signals [8]. A ball seal leakage may cause increased flow rate through the value for the same actuator input, causing the fault vector parameter $f_{0}$ to have a nonzero component associated with the faulty valve. The value of this nonzero element yields the amount of leakage. A shaft seal leakage may cause a diaphragm rupture and consequently a stuck valve. This would cause those elements of $F$ and $f_{n o}$ associated with the faulty valve to change from a value of one to a value of zero and from a value of zero to a nonzero value respectively. A broken wire in the RVDT system may lead to a signal enor, causing the valve to continuously increase its opening until it is fully 


\begin{tabular}{|c|c|c|c|c|}
\hline \multirow{2}{*}{$\begin{array}{l}\text { ACTUATION } \\
\text { FAILUURE MODE }\end{array}$} & \multicolumn{4}{|c|}{ FAULT PARAMETERS } \\
\hline & Fa11 & Fa22 & $1 a 01^{7 *}$ & fao2 \\
\hline NORMAL & 1 & 1 & 0 & 0 \\
\hline OPOV VALVE BALL SEAL LEAKAGE & 1 & 1 & $c_{1}^{+}$ & 0 \\
\hline FPOV VALVE BALL SEAL LEAKAGE & 1 & 1 & 0 & $\mathrm{C}_{2}$ \\
\hline $\begin{array}{l}\text { FPOV and OPOV VALVE BALL } \\
\text { SEAL LEAKAGE }\end{array}$ & 1 & 1 & $c_{1}$ & $\mathrm{c}_{2}$ \\
\hline OPOV BLOCKAGE & $\therefore$ & $\therefore$ & ---1 & $\ldots$ \\
\hline FPOV BLOCKAGE & 1 & 1 & 0 & $-\mathrm{c}_{2}$ \\
\hline $\begin{array}{l}\text { OPOV VALVE BALL SEAL LEAKAGE } \\
\text { FPOV BLOCKAGE }\end{array}$ & 1 & 1 & $-c_{1}$ & $\cdot \mathrm{c}_{2}$ \\
\hline OPOV VALVE STUCK & 0 & 1 & $c_{1}$ & 0 \\
\hline FPOV VALVE STUCK & $\therefore$ & $\therefore$ & 0 & $\mathrm{C}_{2}$ \\
\hline OPOV \& FPOV VALVE STUCK & 0 & 0 & $c_{1}$ & $\mathrm{c}_{2}$ \\
\hline OPOV RVDT FAILURE & 0 & 1 & $c_{1}$ & 0 \\
\hline FPOV RVDT FALLURE & 1 & 0 & 0 & $c_{2}$ \\
\hline OPOV and FPOV RVDT FAlLURE & 0 & 0 & $c_{1}$ & $\mathrm{c}_{2}$ \\
\hline
\end{tabular}

- Fa11 indicates the 1,1 element of the Fa matrix

* fao 1 indicates the 1 st element of the fao vector

$+C_{1} \& C_{2}$ represent the bias terms

Tabel 1 SSME Actuator Fault Signatures

open. Table 1 describes part of these fault signatures, i.e., it gives the values of the fault parameters corresponding to each signature as well as the values for some combinations of these faults.

A complete nonlinear digital transient model (DTM) of the SSME was developed by Rocketdyne Division of Rockwell International Corporation [12]. This nonlinear simulation is used to simulate the SSME dynamic responses for nonimal operation and fault conditions. The inputs of the simulation are the positions of the oxidizer prebumer oxidizer valve (OPOV), and fuel prebumer oxillizer valve (FPOV). The measured simulation outputs are the chamber inlet pressure ( $P c)$, mixture ratio (MR), high pressure fuel turbine speed (SF2), and high pressure oxidizer turbine speed ( $\mathrm{SO} 2$ ). The operating condition selected for study is at $100 \%$ rated power level with nominal mixture ration of 6.026. A closed loop control (PI controller) in the DTM simulation is also active to simulate the actual operation. The sampling time of the system identification is 0.04 second. Pseudo random binary sequences (PRBS) with a magnitude of $1 \%$ of the control command are superimposed on the command signal. A recursive parameter identification scheme is used to identify the fault parameters for all the cases below. In all the following cases, the simulation was started from steady state.

\section{Case 1: OPOV Sruck at Time $=1.0$ Second}

Figure 2 shows a case in which the OPOV stuck at time at 1.0 second. In this case the valve stopped responding to the input command. The expected parameter values for this type of fault are $F_{\mathfrak{u}} I 1=0, F_{a} 22=1, f_{a v} 1=C_{\text {wlas }}$ (the magnitude of bias depends on the valve stuck position and the desired position of the operating condition) and $\mathrm{f}_{\mathrm{ac}} 2=0$. Terminology used to label fault parameters are $F_{a}(1, I)=F_{a} I l, f_{u o}(I)=f_{a v} I$, etc. The simulation shows that the diagnostic system is not only able to iclentify the correct actuator fatult type after the initial transient but also able to estimate the magnitude of the bias due to the fault which can be very important in designing the control accommodation for the fault. Figure 3 shows the on-line calculated residual defined by equation (10) under the hypothesis of an actuator fault. Values of the residual vector

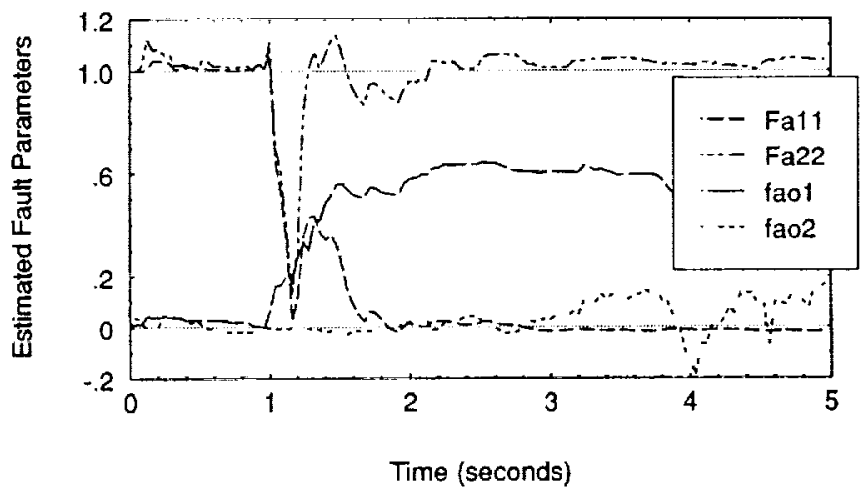

Figure 2 Estimated Faull Parameters in Case 1

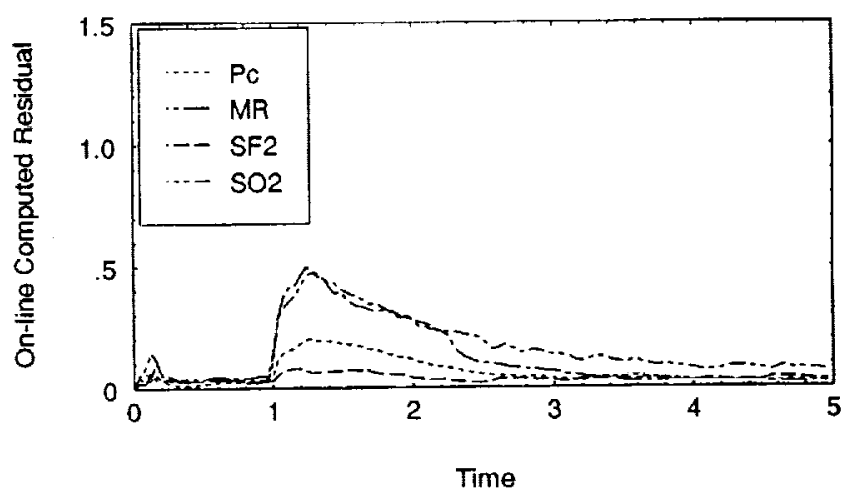

Figure 3 Residual Vector Compuled by the Module Hypothesizing an Actuator Fault in Case 1

return to approximately zero after the initial transient. Figure 4 shows the residual values calculated by the module which hypothesizes system degradation faults. In this figure, the resiclual vector elements are at least ten times higher than those in figure 3. Similarly large residual values were computed by the third module. It can be seen that these values can be used to test the validity of the hypothesis modules. 


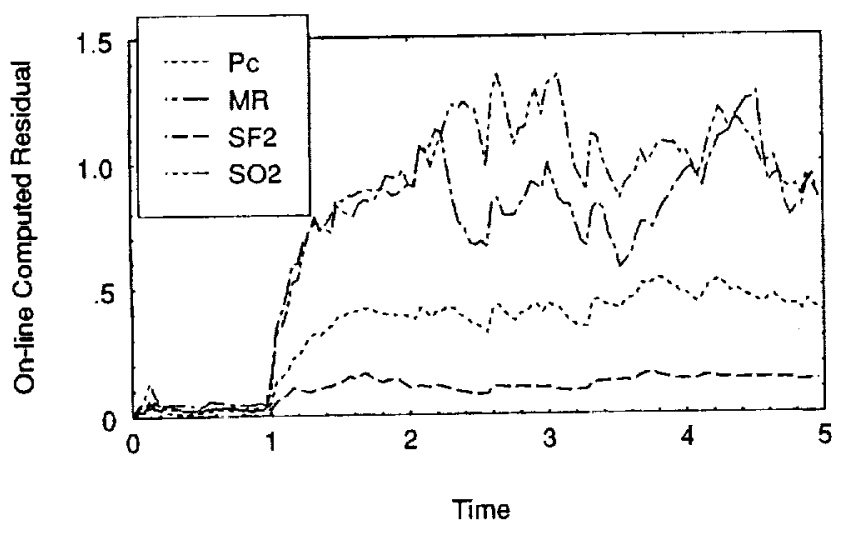

Figure 4 Residual Vector Compuled by the Module Hypothesizing a System Degradation in Case 1

Case 2: OPOV Ball Seal Leak at Time $=1.0$ second

Figure 5 shows a case where an OPOV ball seal leak occurred at time 1.0 second. During the steady state operation before the fault occurs, the fault parameter estimates are $F_{a}=$ $\mathrm{I}$ and $\mathrm{f}_{\mathrm{av}} \mathrm{I}=0$ as expected. After the fault, the parameter values are estimated at $\mathrm{F}_{\mathrm{w}}=1, \mathrm{f}_{\mathrm{us}} \mathrm{l}=2(\%)$ and $\mathrm{f}_{\mathrm{au}} 2=0$. The simulation shows that the diagnostic system is able to identify the correct parameter after the initial transient.

\section{Case 3: FPOV Line Blockage at Time $=1.0$ second}

Figure 6 shows a case in which the FPOV line became blocked at time 1.0 second. The fault parameter estimates start at the correct values of $F_{a}=I$ and $f_{a b} l=0$ prior to 1.0 second. After the fault, the parameter values are estimated at $F_{\mathrm{s}}=I, \mathrm{f}_{\mathrm{ac}} \mathrm{I}$ $=0$ and $f_{u v} 2=-2$. The simulation shows that the diagnostic system is able to identify the correct parameter after the initial transient.

\section{Case 4: Simultaneous OPOV Leakage and FPOV Blockage}

In this case, both Case 1 and 2 faults were introduced at the same time $(T=1.0)$. The final true parameter values in this case are $F_{a}=I, f_{a 0} l=+2.0$ and $f_{a o} 2=-2.0$. Figure 7 shows that the proposed hypothesis testing module is able to correctly estimate the fault parameter values within 2 to 3 seconds.

\section{Case 5: Bias in Chamber Pressure (Pc) Sensor}

Figure 8 shows the results obtained for the case of a faulty sensor with a bias of $1 \%$ on sensor one (chamber pressure). As expected, the results are that the estimated fault parameters $F_{1} \approx I$ and the bias terms $f_{s o} \approx 0$ except $f_{s o} I$ which is the indicator of Pc measurement bias.

As illustrated in these simulation results, both the faultdetection and the estimation of the extent of faults can be determined by using the proposed approach. These simulations indicate that a duration of two seconds is sufficient for the fault detection and diagnosis.

\section{CONCLUSION}

A fault-detection and diagnosis system based on distributed, fault-parameter estimation is developed. Actuator, sensor and system degradation fault modes are considered by the developed FDD system. In the FDD system, the system inputs and outputs are first processed by a series of hypothesis testing modules. Each hypothesis module generates estimates of selected fault parameters and corresponding residuals. The fault parameters and residuals generated by the hypothesis modules are used for fault-detection and diagnosis. The proposed FDD system is demonstrated by applying it to detect

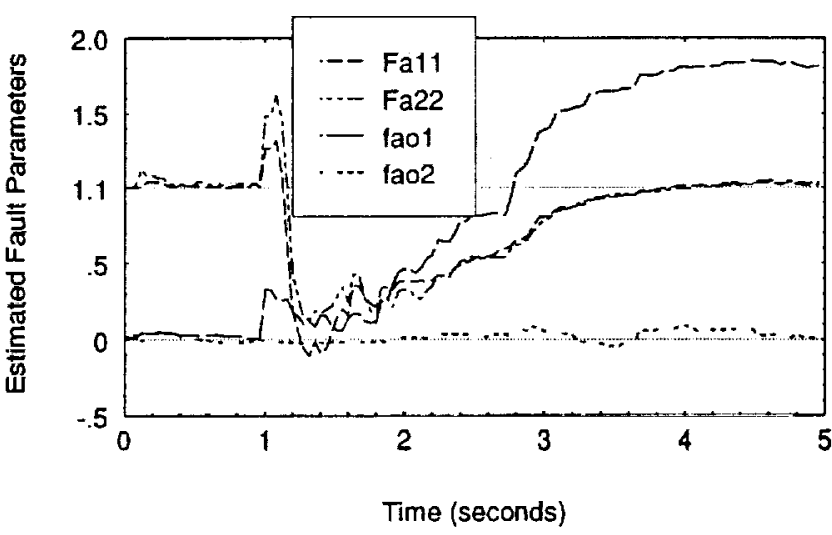

Figure 5 Estimated Fault Parameters in Case 2

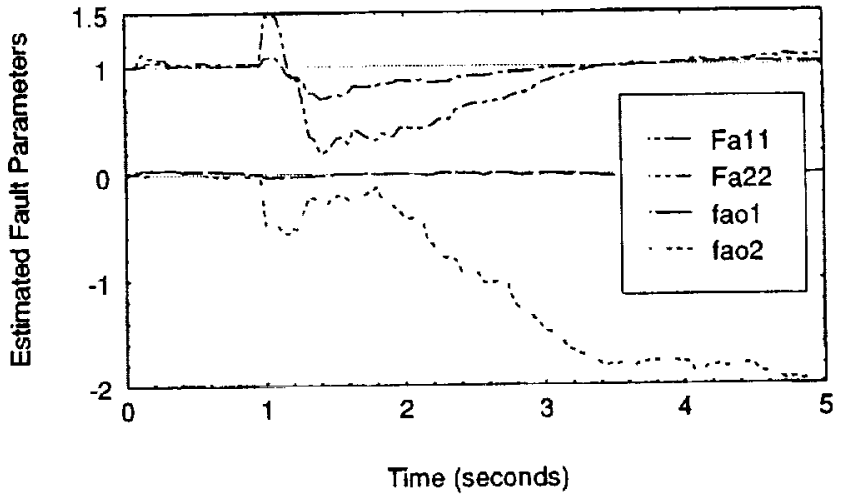

Figure 6 Estimated Fault Parameters in Case 3

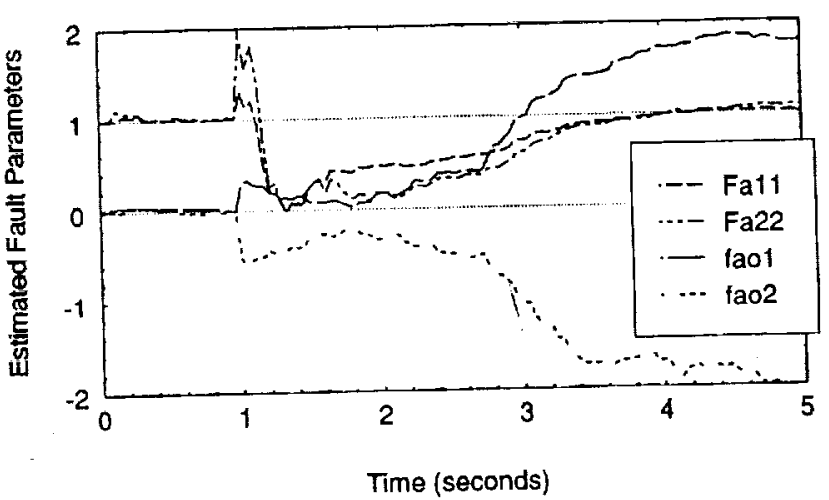

Figure 7 Estimated Fault Parameters in Case 4

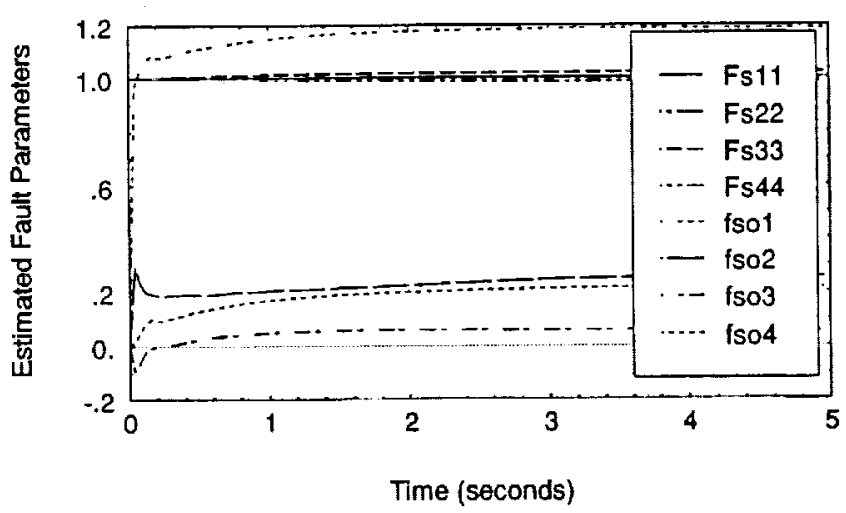

Figure 8 Estimated Fault Parameters in Case 5 
actuator and sensor faults added to a simulation of the Space Shuttle Main Engine. The simulation results show that the proposed FDD system can adequately detect the faults and estimate their magnitudes. Further research in the application of this scheme to system degradation faults is currently underway.

\section{REFERENCES}

1. Guo, T-H. and Merrill W. C., "Distributed Artificial Intelligence Architecture of a Real-Time Diagnostic System for a Reusable Rocket Engine," NASA TM to be published.

2. Merrill, W, C. and Lorenzo, C. F., "A Reusable Rocket Engine Intelligent Control," NASA TM 100963, ALAA-883114 , July 1988.

3. Willsky, A. S., "A Survey of Design Methods for Fault Detection in Dynamic Systems," Automatica, Vol. 12, pp. $601-611,1976$.

4. Isemann, R., "Process Fault Detection Based on Modelling and Estimation Methods," Automatica, Vol. 20, pp. 387 $404,1984$.

5. Gertler, J. J., " Survey of Model-Based Fault Detection and Isolation in Complex Plants," IEEE Control Systems Magazine, pp. 3-1 I, December 1988.

6. Frank, P. M., "Fault Diagnosis in Dynamic Systems Using Analytical and Knowledge-Based Redundancy - A Survey and Some New Results," Automatica, Vol. 26, NO. 3, pp. 459-474, 1990.

7. Eldem, V. and Duyar, A., "Identification of Discrete-Time Multivariable Systems: A Parametrization via $\alpha$-Canonical Form," Submitted to Automatica, June 1989.

8. Tischer, A.E . and Glover, R. C., "Studies and Analyses of the Space Shuttle Main Engine," Prepared for NASA George C. Marshall Space Flight Center, Contract No. NASw-3737, December 1987.

9. Duyar, A., Guo, T-H. and Merrill, W. C., "Space Shuttle Main Engine Model Identification," IEEE Control Systems Magazine, Vol. 10, No. 4, pp. 59-65, 1990.

10. Duyar, A., Eldem, V., Merrill, W. C. and Guo, T-H., "State Space Representation of the Open Loop Dynamics of the Space Shuttle Main Engine," Submitted to ASME J-DSMC, November 1989.

11. Duyar, A., Eldem, V., Merrill, W. C. and Guo, T-H., "A Simplified Dynamic Model of Space Shuttle Main Engine," submitted to ASME Joumal of Dynamic Systems Measurement and Control, June 1990.

12. Rockwell Intemational Corporation, "Engine Balance and Dynamic Model," Report FSCM No. 02602, Spec. No. RL00001., 1981 . 





